\title{
The global dark halo structure of the Andromeda galaxy
}

\author{
Kohei Hayashi and Masashi Chiba \\ Astronomical Institute, Tohoku University, 6-3 Aoba, Sendai 980-8578, Japan \\ email: k.hayasi@astr.tohoku.ac.jp \\ email: chiba@astr.tohoku.ac.jp
}

\begin{abstract}
We set new limits on the global shape of the dark halo in the Andromeda galaxy based on axisymmetric mass models constructed by Hayashi \& Chiba (2012). This is motivated by the fact that CDM models predict non-spherical virialized dark halos, which reflect the process of mass assembly in the galactic scale. Based on the application of our models to latest kinematical data of globular clusters and dwarf spheroidal galaxies in the Andromeda halo, we find that the most plausible cases for Andromeda yield not a spherical but a prolate shape for its dark halo. We also find that the prolate dark halo is consistent with theoretical predictions in which the satellites are distributed anisotropically and preferentially located along major axes of their galactic host halos. It is a reflection of the intimate connection between galactic dark matter halos and the cosmic web.
\end{abstract}

Keywords. Andromeda galaxy, Kinematics and dynamics, Local Group, Dark matter

\section{The data and model}

We use a sample of $91 \mathrm{GCs}$ and $15 \mathrm{dSphs}$ in the halo region of M31. For the sample of GCs, we adopt the Revised Bologna Gatalogue of M31 GCs, while for the sample of dSphs, we use published data in Tollerud et al. (2012). For the density profile of these halo tracers, we assume a power law form, $\Sigma_{*}\left(m_{*}^{\prime}\right) \propto m_{*}^{\prime \gamma}$, where $m_{*}^{\prime 2}=x^{2}+y^{2} / q^{\prime 2}, q^{\prime}$ is the projected axial ratio. We obtain $\gamma=-3.00 \pm 0.05$ and $q^{\prime} \simeq 1.18 \pm 0.20$, respectively. For the dark matter halo, we assume the power-law form $\rho(R, z)=\rho_{0}\left(\mathrm{~m} / \mathrm{b}_{\text {halo }}\right)^{-\alpha}[1+$ $\left.\left(m / b_{\text {halo }}\right)^{2}\right]^{-\delta}$, where $m^{2}=R^{2}+z^{2} / Q^{2}, Q$ is an axis ratio, $b_{\text {halo }}$ is a scale length and $\rho_{0}$ is a density normalization. We then solve the axisymmetric Jeans equations, and determine the most likely halo parameters by fitting to the observed line of sight velocities.

\section{Prolate dark halo of the Andromeda galaxy}

Based on the maximum likelihood analysis, we find that the most plausible cases for Andromeda yield a prolate shape for its dark halo, irrespective of assumed dark matter density profiles. Furthermore, the prolate dark halo is consistent with theoretical predictions in which the satellites are distributed anisotropically and preferentially located along major axes of their galactic host halos.

\section{References}

Hayashi, K. \& Chiba, M. 2012, ApJ, 755, 145

Tollerud, E. T., et al. 2012, ApJ, 752, 45 